\title{
Dimethyl Sulfoxide Leads to Decreased Osteogenic Differentiation of Stem Cells Derived from Gingiva via Runx2 and Collagen I Expression
}

\author{
Hyunjin Lee ${ }^{1} \quad$ Jun-Beom Park ${ }^{1}$ \\ ${ }^{1}$ Department of Periodontics, College of Medicine, The Catholic \\ University of Korea, Seoul, Republic of Korea
}

Eur J Dent 2019;13:131-136

\begin{abstract}
Address for correspondence Jun-Beom Park, DDS, MSD, PhD, Department of Periodontics, Seoul St. Mary's Hospital, College of Medicine, The Catholic University of Korea, 222, Banpo-daero, Seochogu, Seoul 06591, Republic of Korea (e-mail: jbassoonis@yahoo.co.kr).
\end{abstract}

\begin{abstract}
Objectives Dimethyl sulfoxide (DMSO) plays various functions, including cellular functions such as cellular growth. The aim of this study was to evaluate the effects of DMSO on the proliferation and osteogenic differentiation of human gingiva-derived stem cells.

Materials and Methods Stem cells derived from gingiva were cultured in the presence of DMSO at concentrations ranging from 0.01 to $10 \%$.

Statistical Analysis We performed a one-way analysis of variance (ANOVA) with post hoc test to determine the differences between the groups using a commercially available program and the level of significance was 0.05 .

Results The cells in the control group showed normal fibroblast morphology. The cells treated with $0.01 \%, 0.01 \%, 0.1 \%$, and $1 \%$ DMSO were morphologically similar to those from the control group on each day. Statistically significant decreases in cell counting kit-8 (CCK-8) values were seen in the $3 \%$ and $10 \%$ DMSO groups $(p<0.05)$. A statistically significant decrease in alkaline phosphatase activity was seen in the $3 \%$ DMSO group. $(p<0.05)$. The application of DMSO produced a decrease in alizarin red

Keywords

- osteogenic differentiation

- proliferation

- dimethyl sulfoxide

- gingiva

- stem cells $S$ staining. The expression of Runx 2 and collagen I by immunofluorescence decreased as the dose of lovastatin increased.

Conclusion The effects of DMSO on the viability of osteogenic differentiation among stem cells derived from human gingiva were evaluated. Applying DMSO produced decreased cell viability and decreased osteogenic differentiation in this experimental setting. This should be considered when designing and interpreting the data, and a DMSO-free method may be considered for bone regeneration applications.
\end{abstract}

\section{Introduction}

Dimethyl sulfoxide (DMSO) is widely applied as a solvent for molecules, making them soluble in organic media as well as aqueous media. ${ }^{1}$ DMSO is shown to play various functions, including in cellular growth and cellular functions. ${ }^{2}$ DMSO is known to affect mitochondrial integrity and membrane potential. ${ }^{3}$ Also, DMSO may produce apoptosis in the developing central nervous system. ${ }^{4}$ DMSO inhibited inflammation in experimental models and affected intestinal cytokine production. ${ }^{5}$ Although DMSO is widely used for the preservation of liquid nitrogen-frozen cells, there are possibilities associated with toxicity in the transplantion. ${ }^{6,7}$

In a previous study, our group isolated and characterized stem cells from human gingival connective tissue. ${ }^{8}$ The potential differentiation ability of gingiva-originated human 
mesenchymal stem cells was tested, as was the feasibility of application in tissue-engineering purposes. ${ }^{9}$ However, the effects of DMSO on stem cells have not yet been widely demonstrated. ${ }^{10}$ Thus, the aim of this study is to evaluate the effects of DMSO on the proliferation and osteogenic differentiation of human gingiva-derived stem cells. The alkaline phosphatase activity test and alizarin red S staining were used to assess the differentiation and mineralization of the treated cells. Quantitative real-time polymerase chain reaction was used to evaluate the mRNA levels of Runt-related transcription factor 2 (Runx2) and collagen I, and the protein expressions of Runx2 and collagen I were measured using Western blot analysis. To our knowledge, this investigation is the first to elucidate the effects of DMSO on the expressions of Runx2 and collagen I in mesenchymal stem cells derived from gingiva.

\section{Materials and Methods}

\section{Stem Cells Derived from Human Gingiva}

We collected the gingiva of healthy patients visiting the Department of Periodontics, Seoul St. Mary's Hospital, College of Medicine, Catholic University of Korea. The Institutional Review Board reviewed and approved the study (KC11SISI0348), and informed consent was obtained from the participants. All the methods were performed in accordance with the relevant guidelines and regulation. The obtained tissue was placed in sterile phosphate-buffered saline (PBS; Welgene, Daegu, South Korea) containing $100 \mathrm{U} / \mathrm{mL}$ penicillin and $100 \mu \mathrm{g} / \mathrm{mL}$ streptomycin (Sigma-Aldrich Co., St. Louis, MO, United States). We removed the epithelium of the obtained tissue and minced the tissue into 1 to $2 \mathrm{~mm}$ fragments. We digested the tissue with media containing collagenase IV (Sigma-Aldrich Co.). The cells were incubated in an environment with $5 \% \mathrm{CO}_{2}$ and $95 \% \mathrm{O}_{2}$ at $37^{\circ} \mathrm{C}$. Cells that were not attached to the culture dish were removed, and the media was changed every 2 to 3 days.

\section{Evaluation of Cellular Morphology}

We plated the cells at a density of $2.0 \times 10^{3}$ cells/well in 96-well plates. We incubated the cells in osteogenic media $(\alpha-$ minimal essential medium [ $\alpha$-MEM, Gibco, Grand Island, NY, United States]) supplemented with $15 \%$ fetal bovine serum (FBS, Gibco), 200 mM L-glutamine (Sigma-Aldrich Co.), $10 \mathrm{mM}$ of ascorbic acid 2-phosphate (Sigma-Aldrich Co.), $38 \mu \mathrm{g} / \mathrm{mL}$ of dexamethasone, $2 \mathrm{mg} / \mathrm{mL}$ of glycerophosphate disodium salt hydrate, and $100 \mathrm{U} / \mathrm{mL}$ penicillin, and $100 \mu \mathrm{g} / \mathrm{mL}$ streptomycin (Sigma-Aldrich Co.) in the presence of DMSO (Sigma-Aldrich Co.) at final concentrations ranging from 0 (control) to $0.01 \%$ (low dose), $0.1 \%$ (medium dose), $1 \%$ (high dose), 3\% (super), and 10\% (extreme). We used the inverted microscopy (CKX41SF; Olympus Corporation, Tokyo, Japan) to evaluate the morphology of the tested cells at days $1,3,5,7$, and 10 .

\section{Determination of Cell Viability}

We tested the viability of the cells on days 1, 3, 5, and 7 using the counting kit-8 (CCK-8) assay. We added tetrazolium monosodium salt (CCK-8; Dojindo, Tokyo, Japan) to the culture, and we incubated the cells at $37^{\circ} \mathrm{C}$ for 2 hours. We used a microplate reader (BioTek Instruments Inc., Winooski, VT, United States) to find the spectrophotometric absorbance at $450 \mathrm{~nm}$. The tests were performed in triplicate.

\section{Alkaline Phosphatase Activity Assays}

We performed alkaline phosphatase activity assays on days $3,7,10,14$, and 21 . We used trypsin (Gibco) to detach the cells, and used a commercially available kit (K412-500, BioVision, Inc., Milpitas, CA, United States) to evaluate alkaline phosphatase activity assays. We used a microplate reader (BioTek Instruments Inc.) to measure the spectrophotometric absorbance of the samples.

\section{Evaluation of Alizarin Red S Staining}

We performed alizarin red S staining on days 5, 7, and 21 . We washed, fixed, and stained the cells with $2 \%$ alizarin red S solution (ScienCell Research Laboratories, Inc., Carlsbad, CA, USA) and evaluated the cells with a microscope (CKX41SF, Olympus Corporation). We removed nonspecifically bound stains, and solubilized bound dye with $10 \%$ cetylpyridinium chloride (Sigma-Aldrich Co.). We performed spectrophotometric quantification at $560 \mathrm{~nm}$ (BioTek Instruments Inc.).

\section{Immunofluorescence}

We performed an immunofluorescent assay for Runx2 (ab76956, Abcam, Cambridge, United Kingdom) and collagen I (ab6308, Abcam) on days 1, 3, 5, and 7. We fixed, permeabilized, blocked, and incubated the cells with primary antibodies. We incubated the cultures with fluorescein isothiocyanate-conjugated secondary antibody (F2761, Abcam); then, stained the washed cells with 4', 6-diamidino-2-phenylindole (DAPI). We analyzed the cells using a fluorescence microscope (Axiovert 200, Zeiss, Jena, Germany).

\section{Total RNA Extraction and Quantification by Real-Time Polymerase Chain Reaction}

We isolated total RNA from the cultured cells on day 11 using a GeneJET RNA Purification Kit (Thermo Fisher Scientific, Inc., Waltham, MA, United States). We determined quantities by spectrophotometer (ND-2000, Thermo Fisher Scientific, Inc., Wilmington, DE, United States) with ratios of absorbance at 260 and $280 \mathrm{~nm}$.

We designed the sense and antisense primers based on GenBank. The primer sequences were as follows: Runx-2 Forward 5': AAT GAT GGT GTT GAC GCT GA - 3'; Reverse 5': TTG ATA CGT GTG GGA TGT GG - 3'; Collagen I Forward 5': TCA TGG CCC TCC AGC CCC CAT3'; and Reverse 5': ATG CCT CTT GTC CTT GGG GTT C - 3'. $\beta$-actin served as a housekeeping gene for normalization. We detected mRNA expression by RT-PCR using SYBR Green Real-Time PCR Master Mixes (Enzynomics, Daejeon, South Korea) based on the manufacturer's protocol. We performed the experiments in triplicate.

\section{Western Blot Analysis}

We washed and solubilized the cells using lysis buffer (Thermo Fisher Scientific, Inc., Waltham, MA, United States) 
containing protease and phosphatase inhibitor cocktail (Thermo Fisher Scientific, Inc.) as well as phenylmethylsulfonyl fluoride (Sigma-Aldrich Co.) on day 10 . We quantified the lysates using the BCA assay (Thermo Fisher Scientific, Inc.). We separated protein samples using sodium dodecyl sulfate polyacrylamide gel electrophoresis and then transferred for immunoblotting. We incubated the membranes with the primary antibodies overnight at $4^{\circ} \mathrm{C}$, and then with the secondary antibody for 1-hour at room temperature. The antibodies included those against Runx2, collagen I, and glyceraldehyde 3-phosphate dehydrogenase (GAPDH), as well as secondary antibodies linked with horseradish peroxidase were used. We purchased the antibodies from Abcam (Cambridge, United Kingdom) and BD Bioscience (San Jose, CA, United States).

\section{Statistical Analysis}

We presented the data as the means \pm standard deviations of the experiments. We used the Shapiro-Wilk test to test for normality. We performed a one-way analysis of variance (ANOVA) with post hoc test to determine the differences between the groups using a commercially available program (SPSS 12 for Windows, SPSS Inc., Chicago, IL, United States). The level of significance was 0.05 .

\section{Results}

\section{Cellular Morphology and Cell Viability of Stem Cells Grown in Osteogenic Media}

The shape of cells grown in osteogenic media on days 1, 3, 5, 7, and 10 are shown in - Fig. 1A and Supplementary - Fig. 1A-D (available online only). The cells treated with $0.01 \%, 0.01 \%$, $0.1 \%$, and $1 \%$ DMSO were morphologically similar to those from the control group on each day. The cells from the 3\% and 10\% groups were significantly different from the others.

The relative values of CCK-8 on days $1,3,5$, and 7 are shown in - Fig. 1B-E. The relative values of CCK-8 at day 5 for the $0.01 \%, 0.10 \%, 1 \%, 3 \%$, and $10 \%$ groups are $105.3 \pm$ $8.0 \%, 120.0 \pm 12.5 \%, 90.1 \pm 4.8 \%, 76.6 \pm 1.3 \%$, and $63.8 \pm 0.7 \%$, respectively, when the control ( $0 \%$ group) at day 1 is considered $100 \%$ (100.0 $\pm 4.7 \%$ ).

\section{Alkaline Phosphatase Activity Assays}

The alkaline phosphatase activity treated with DMSO at days $3,7,10$, and 14 is shown in - Fig. 1F. The absorbance values of cells on day 14 grown with $0 \%, 0.01 \%, 0.10 \%, 1 \%$, and $3 \%$ DMSO were $0.156 \pm 0.005,0.164 \pm 0.002,0.166 \pm 0.001,0.179$ $\pm 0.003,0.112 \pm 0.002$, and $0.089 \pm 0.002$, respectively. The $3 \%$ DMSO group showed a statistically significant decrease in alkaline phosphatase activity on days 10 and 14 , compared with the control group at each time point $(p<0.05)$.

\section{Mineralization Assay}

The results of the alizarin red S staining on days 5, 14, and 21 following treatment with different concentrations of DMSO in osteogenic supplement are shown in - Fig. $2 \mathrm{~A}$ and Supplementary - Fig. 2A (available online only). The absorbance of cells on day 14 grown with $0 \%, 0.01 \%, 0.10 \%, 1 \%$, and $3 \%$ DMSO was $0.071 \pm 0.001,0.089 \pm 0.001,0.081 \pm 0.001$, $0.081 \pm 0.000,0.081 \pm 0.001$, and $0.118 \pm 0.002$, respectively (-Fig. 2B). The absorbance of cells on day 21 grown with $0 \%$,
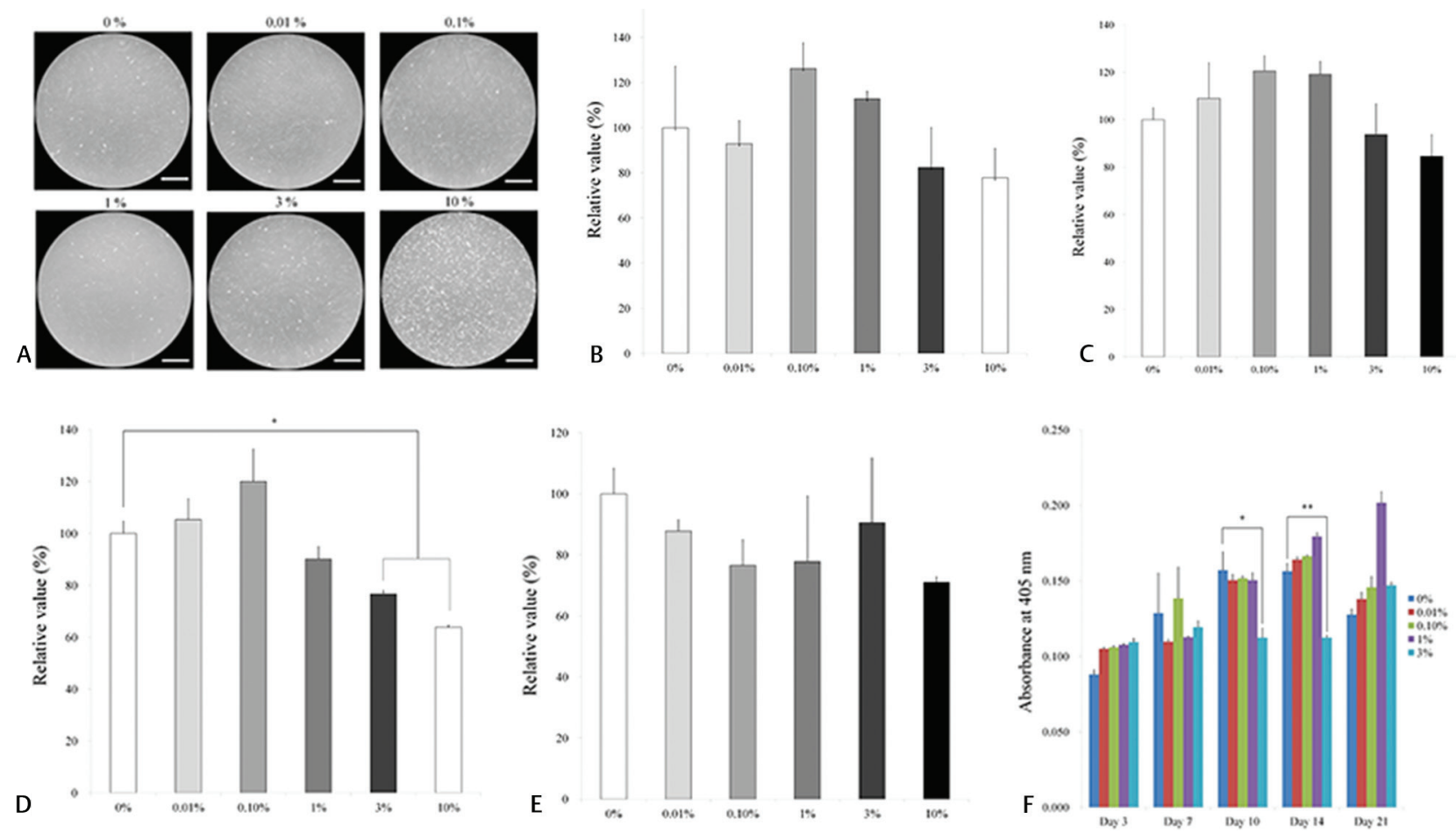

Fig. 1 Evaluation of cell morphology, viability, and alkaline phosphatase activity. (A) Evaluation of cell morphology on day 1 using inverted microscopy following treatment with different concentrations of dimethyl sulfoxide $(0 \%, 0.01 \%, 0.1 \%, 1.0 \%, 3.0 \%$, and $10.0 \%)$ on osteogenic supplement. The bar indicates $400 \mu \mathrm{m}$. (B) The CCK-8 assay results on day 1 cultured with osteogenic media. (C) The CCK-8 assay results on day 3. (D) The CCK-8 assay results on day 5. (E) The CCK-8 assay results on day 7. (F) Alkaline phosphatase activity on days $3,7,10,14$, and 21. CCK-8, cell counting kit-8. 
$0.01 \%, 0.10 \%, 1 \%$, and $3 \%$ DMSO was $0.183 \pm 0.001,0.150 \pm$ $0.002,0.170 \pm 0.001,0.159 \pm 0.003,0.149 \pm 0.006$, and 0.166 \pm 0.002 , respectively.

\section{Validation of mRNA Expression by Real-Time Polymerase Chain Reaction}

The quantitative RT-PCR results for the mRNA levels of Runx2 and collagen I are shown in - Fig. 2C, D. The relative expressions of Runx2 in osteogenic media at day 11 among the $0 \%, 0.01 \%, 0.10 \%$, and $1 \%$ groups were $100.0 \pm 50.8 \%, 293.4$ $\pm 156.2 \%, 157.0 \pm 80.2 \%$, and $146.3 \pm 140.8 \%$, respectively
( $\mathbf{- F i g . ~ 2 C ) . ~ T h e ~ r e l a t i v e ~ e x p r e s s i o n s ~ o f ~ c o l l a g e n ~ I ~ i n ~ o s t e o g e n - ~}$ ic media at day 11 among the $0 \%, 0.01 \%, 0.10 \%$, and $1 \%$ groups were $100.0 \pm 18.8 \%, 82.1 \pm 41.9 \%, 74.3 \pm 35.2 \%$, and $217.1 \pm$ $36.5 \%$, respectively ( - Fig. 2D).

\section{Immunofluorescence}

The immunofluorescent assays for Runx2 and collagen I for days $1,3,5$, and 7 are shown in -Fig. $\mathbf{3 A}$, B and Supplementary - Figs. 3 and $\mathbf{4}$ (available online only). No significant change in Runx2 expression was noted as the dose of DMSO increased. The expression of collagen I seemed to show
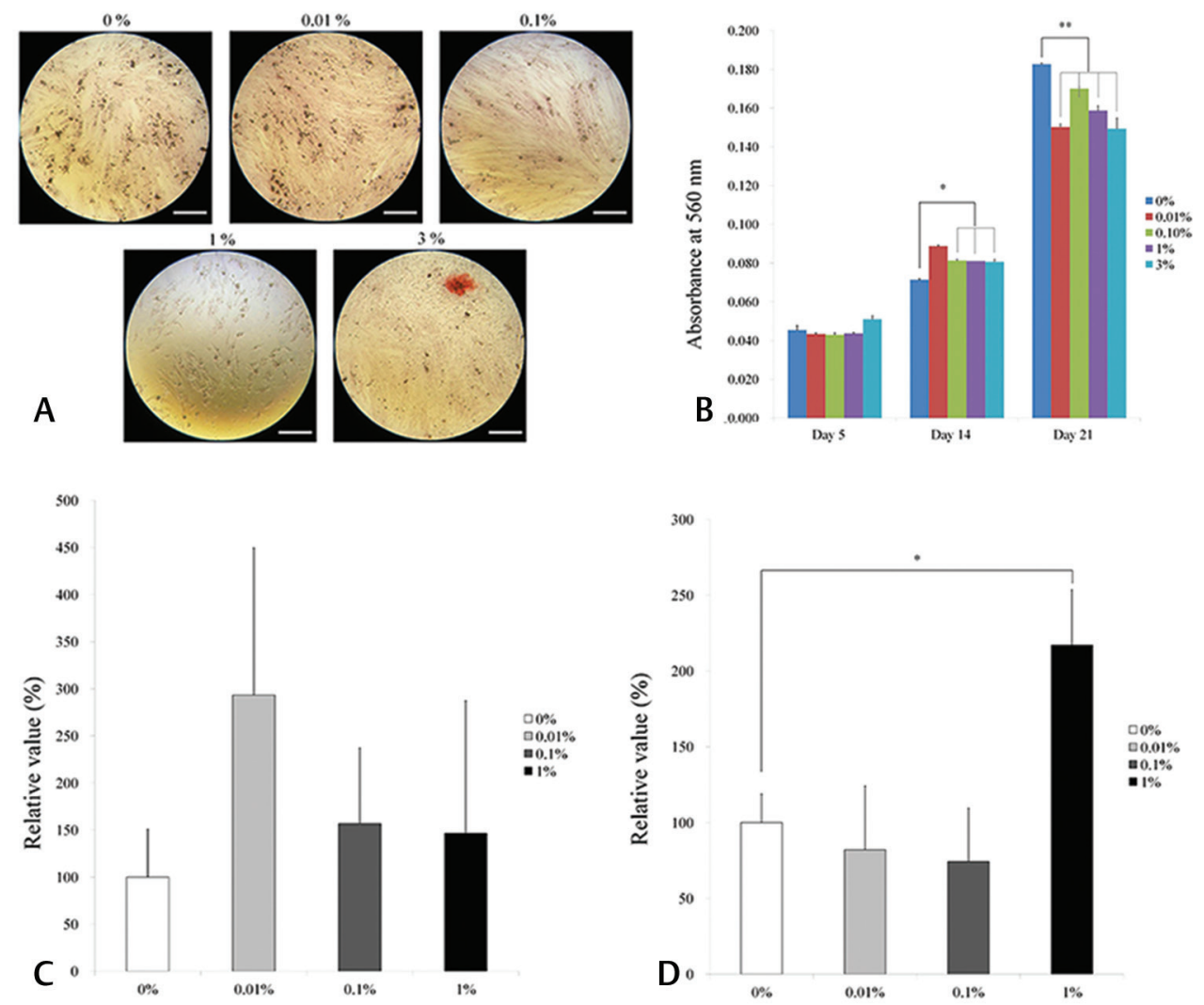

Fig. 2 Alizarin red S staining and quantitative RT-PCR results. (A) Results of alizarin red S staining on day 5 following treatment with different concentrations of dimethyl sulfoxide ( $0 \%, 0.01 \%, 0.1 \%, 1.0 \%$, and 3.0\%) on osteogenic supplement. The bar indicates $200 \mu \mathrm{m}$. (B) Quantitative results of alizarin red S staining on days 5, 14, and 21. (C) Quantitative RT-PCR results of Runx2 expression on day 11 on osteogenic media. (D) Quantitative RT-PCR results of collagen I expression on day 11 on osteogenic media. RT-PCR, real-time polymerase chain reaction.

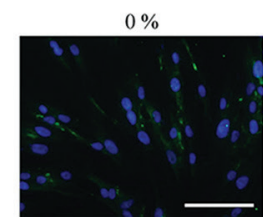

$1 \%$

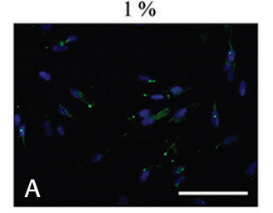

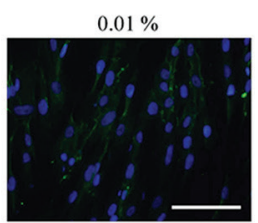

$3 \%$

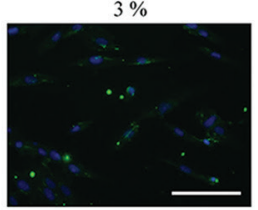

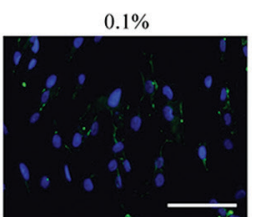

$10 \%$

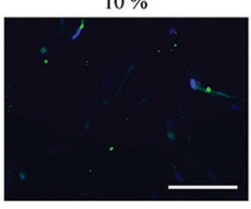

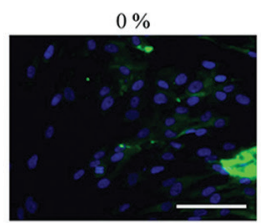

$1 \%$

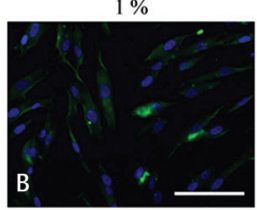

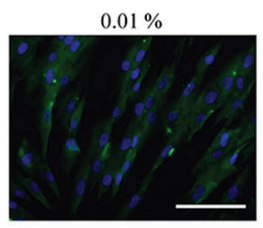

$3 \%$

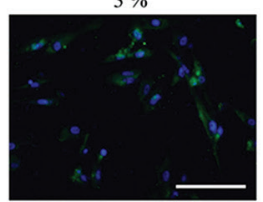

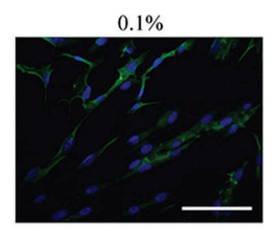

$10 \%$

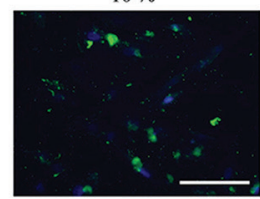

Fig. 3 Immunofluorescence results of Runx2 and collagen I. (A) Immunofluorescence results of Runx2 on day 1 . The bar indicates $200 \mu$ m. (B) Immunofluorescence results of collagen I on day 1 . The bar indicates $200 \mu \mathrm{m}$. 


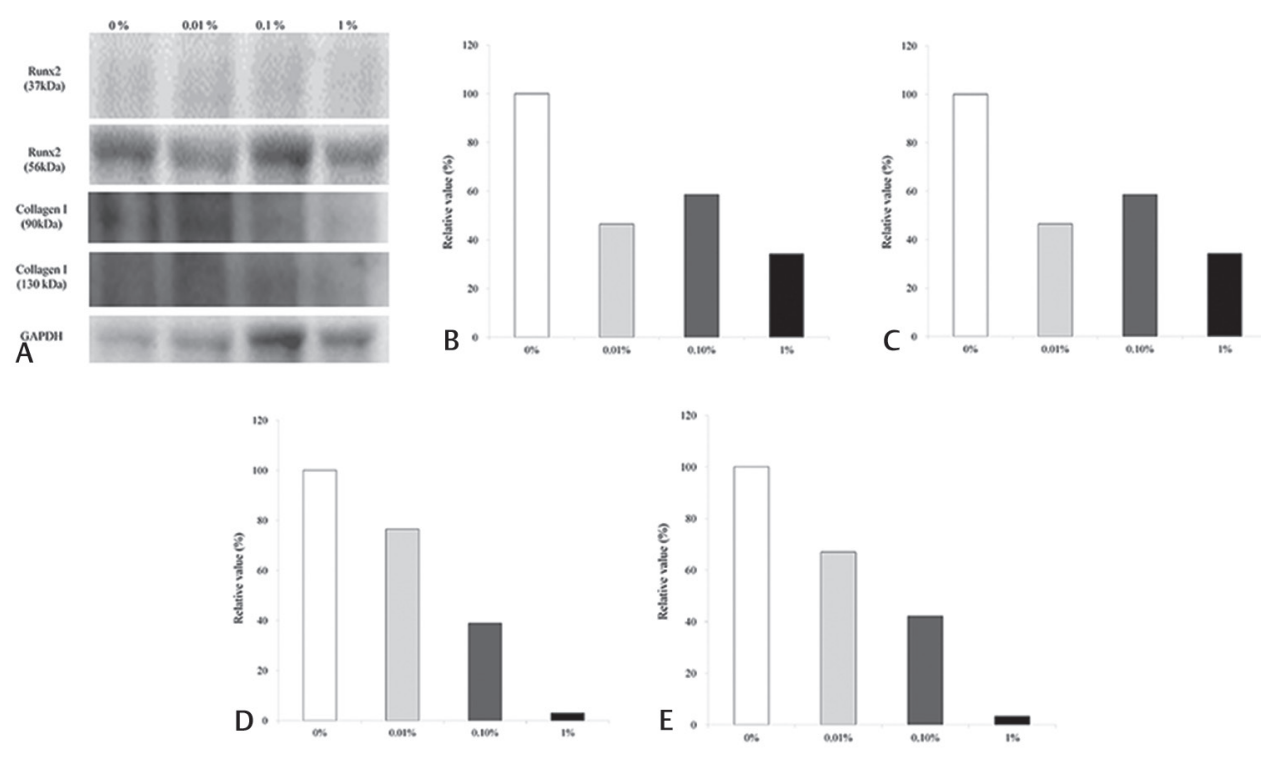

Fig. 4 Western blot analysis to detect the protein expression of Runx2 and collagen I at day 10 and quantitative analysis of the protein expression of Runx2 and collagen I after normalization with GAPDH levels by densitometry. (A). Western blot analysis results. (B) Runx2 (37 kDa) expression on osteogenic media. (C) Runx2 (56 kDa) expression on osteogenic media. (D) Collagen I (90 kDa) expression on osteogenic media. (E) Collagen I (130 kDa) expression on osteogenic media. GAPDH, glyceraldehyde 3-phosphate dehydrogenase.

similar trends: there were no significant changes as the dose of DMSO increased.

\section{Western Blot}

Western blot analysis was performed to detect the protein expression of Runx2, collagen I, and GAPDH at day 10 ( - Fig. 4). The relative expressions of Runx2 $(37 \mathrm{kDa})$ in osteogenic media at day 10 among the $0 \%, 0.01 \%, 0.10 \%$, and $1 \%$ groups were $100.0 \%, 46.4 \%, 58.6 \%$, and $34.2 \%$, respectively. The relative expressions of Runx2 (56 kDa) in osteogenic media at day 10 among the $0 \%, 0.01 \%, 0.10 \%$, and $1 \%$ groups were $100.0 \%, 119.7 \%, 165.3 \%$, and 193.8 , respectively. The relative expressions of collagen I (90 $\mathrm{kDa}$ ) in osteogenic media at day 10 among the $0 \%, 0.01 \%$, $0.10 \%$, and $1 \%$ groups were $100.0 \%, 76.5 \%, 38.9 \%$, and $3.0 \%$, respectively. The relative expressions of collagen I (130 $\mathrm{kDa}$ ) in osteogenic media at day 10 among the $0 \%, 0.01 \%$, $0.10 \%$, and $1 \%$ groups were $100.0 \%, 66.9 \%, 42.1 \%$, and $3.3 \%$, respectively.

\section{Discussion}

We tested the effects of DMSO on gingiva-derived stem cells, which clearly showed that DMSO in the tested concentrations decreased the osteogenic differentiation of the stem cells via the Runx2 and collagen I pathways.

In this study, cellular viability was determined using CCK8 assays. The viability may be determined using membrane leakage assays, mitochondrial activity assays, and functional assays. ${ }^{11}$ Membrane leakage assays using propidium iodide and trypan blue are based on the principle that live cells possess intact membranes, which excludes the dye from going into the cells. ${ }^{12}$ An MTT assay determines the cellular viability based on the mitochondrial activity, but this requires solubilization procedures. ${ }^{13}$ The CCK-8 assay has the advantage of having no requirements for the solubilization procedure, along with lower cytotoxicity. ${ }^{14}$ This report discusses the effects of DMSO on the viability of stem cells under predetermined concentrations ( 0.01 to $10 \%)$. The trend of decreased viability was noted with higher concentrations of DMSO but with statistical significance only in the 3 and $10 \%$ groups.

Osteogenic differentiation may be determined using various methods. ${ }^{8,13}$ Alkaline phosphatase activity is usually considered an early marker of osteogenic differentiation. ${ }^{15}$ This study showed that the alkaline phosphatase activity of the control group increased for up to 14 days, and then a decrease was noted. With its higher concentration, the $3 \%$ group showed a statistically significant decrease in alkaline phosphatase activity. Alizarin red S staining is used to determine the deposition of calcium, which happens after matrix maturation. ${ }^{9}$ Decreased deposition of calcium was noted with the application of DMSO. The statistically significant decrease in calcium deposition was noted in the $0.01 \%$ group up to the $3 \%$ group at day 21 .

This study used qualitative RT-PCR to evaluate mRNA expression and Western blot analysis to determine the protein expression of Runx2 and collagen I. There were no statistically significant changes in the mRNA expression of Runx2 and collagen I. However, the Western blot analysis showed a dose-dependent decrease of protein expression of Runx2 and collagen I. Runx2 is reported to be a prerequisite for osteogenic differentiation, ${ }^{16}$ and collagen I is known to be involved in regulating osteoblast phenotypes. ${ }^{17}$

The adverse effects of DMSO on stem cells should be considered when designing and interpreting the data. Infusion of DMSO may lead to side effects, including nausea, vomiting, fever, anaphylaxis, and headache. ${ }^{18}$ In this regard, it has been reported that manual single wash reduces the frequency of adverse effects, but since the procedure is time-consuming, this is only recommended for patients with high risk of DMSO 
toxicity. ${ }^{19}$ Moreover, a previous method involves the use of an automatic wash method for removing dimethyl sulfoxide in autologous hematopoietic stem cell transplantation, which is reported to decrease the adverse effects related to infusion. ${ }^{20}$ It was suggested that DMSO-free processing may be a safe protocol for banking and manufacturing stem cells, especially for regeneration applications. ${ }^{21,22}$

\section{Conclusions}

The effects of DMSO on the viability of osteogenic differentiation among stem cells derived from human gingiva were evaluated. Applying DMSO decreased cell viability and osteogenic differentiation in this experimental setting. This should be considered when designing and interpreting the data, and DMSO-free methods may be considered for bone regeneration applications.

\section{Funding}

This research was supported by Research Fund of Seoul St. Mary's Hospital, The Catholic University of Korea.

\section{Conflict of Interest}

None declared.

\section{References}

1 Santos NC, Figueira-Coelho J, Martins-Silva J, Saldanha C. Multidisciplinary utilization of dimethyl sulfoxide: pharmacological, cellular, and molecular aspects. Biochem Pharmacol 2003;65(7):1035-1041

2 Pal R, Mamidi MK, Das AK, Bhonde R. Diverse effects of dimethyl sulfoxide (DMSO) on the differentiation potential of human embryonic stem cells. Arch Toxicol 2012;86(4):651-661

3 Yuan C, Gao J, Guo J, et al. Dimethyl sulfoxide damages mitochondrial integrity and membrane potential in cultured astrocytes. PLoS One 2014;9(9):e107447

4 Hanslick JL, Lau K, Noguchi KK, et al. Dimethyl sulfoxide (DMSO) produces widespread apoptosis in the developing central nervous system. Neurobiol Dis 2009;34(1):1-10

5 Li YM, Wang HB, Zheng JG, et al. Dimethyl sulfoxide inhibits zymosan-induced intestinal inflammation and barrier dysfunction. World J Gastroenterol 2015;21(38):10853-10865

6 Morris C, de Wreede L, Scholten M, et al; Chronic Malignancies and Lymphoma Working Parties of EBMT. Should the standard dimethyl sulfoxide concentration be reduced? Results of a European Group for Blood and Marrow Transplantation prospective noninterventional study on usage and side effects of dimethyl sulfoxide. Transfusion 2014;54(10):2514-2522

7 Windrum P, Morris TC, Drake MB, Niederwieser D, Ruutu T; EBMT Chronic Leukaemia Working Party Complications Subcommittee. Variation in dimethyl sulfoxide use in stem cell transplantation: a survey of EBMT centres. Bone Marrow Transplant 2005;36(7):601-603

8 Jin SH, Lee JE, Yun JH, Kim I, Ko Y, Park JB. Isolation and characterization of human mesenchymal stem cells from gingival connective tissue. J Periodontal Res 2015;50(4):461-467

9 Ha DH, Pathak S, Yong CS, Kim JO, Jeong JH, Park JB. Potential differentiation ability of gingiva originated human mesenchymal stem cell in the presence of tacrolimus. Sci Rep 2016;6:34910

10 Qiu Z, Mishra A, Li M, et al. Marmoset induced pluripotent stem cells: Robust neural differentiation following pretreatment with dimethyl sulfoxide. Stem Cell Res (Amst) 2015; 15(1):141-150

11 Lee SI, Yeo SI, Kim BB, Ko Y, Park JB. Formation of size-controllable spheroids using gingiva-derived stem cells and concave microwells: Morphology and viability tests. Biomed Rep 2016;4(1):97-101

12 Yook S, Jeong JH, Jung YS, et al. Molecularly engineered islet cell clusters for diabetes mellitus treatment. Cell Transplant 2012;21(8):1775-1789

13 Park JB, Zhang H, Lin CY, et al. Simvastatin maintains osteoblastic viability while promoting differentiation by partially regulating the expressions of estrogen receptors $\alpha$. J Surg Res 2012;174(2):278-283

14 Jeong SH, Lee JE, Kim BB, Ko Y, Park JB. Evaluation of the effects of Cimicifugae Rhizoma on the morphology and viability of mesenchymal stem cells. Exp Ther Med 2015;10(2):629-634

15 Jin SH, Kweon H, Park JB, Kim CH. The effects of tetracycline-loaded silk fibroin membrane on proliferation and osteogenic potential of mesenchymal stem cells. J Surg Res 2014;192(2):e1-e9

16 Komori T. Regulation of osteoblast differentiation by Runx2. Adv Exp Med Biol 2010;658:43-49

17 Shi S, Kirk M, Kahn AJ. The role of type I collagen in the regulation of the osteoblast phenotype. J Bone Miner Res 1996;11(8):1139-1145

18 González-López TJ, Sánchez-Guijo FM, Ortín A, et al. Ischemic stroke associated with the infusion of DMSO-cryopreserved auto-PBSCs. Bone Marrow Transplant 2011;46(7):1035-1036

19 Akkök CA, Holte MR, Tangen JM, Ostenstad B, Bruserud O. Hematopoietic engraftment of dimethyl sulfoxide-depleted autologous peripheral blood progenitor cells. Transfusion 2009;49(2):354-361

20 Sánchez-Salinas A, Cabañas-Perianes V, Blanquer $\mathrm{M}$, et al. An automatic wash method for dimethyl sulfoxide removal in autologous hematopoietic stem cell transplantation decreases the adverse effects related to infusion. Transfusion 2012;52(11):2382-2386

21 Rogulska O, Petrenko Y, Petrenko A. DMSO-free cryopreservation of adipose-derived mesenchymal stromal cells: expansion medium affects post-thaw survival. Cytotechnology 2016;69(2):265-276

$22 \mathrm{Kim}$ JH, Ko SY, Lee JH, Kim DH, Yun JH. Evaluation of the periodontal regenerative properties of patterned human periodontal ligament stem cell sheets. J Periodontal Implant Sci 2017;47(6):402-415 